\title{
Unusual presentation of chylothorax secondary to a giant abdominal mass
}

\author{
Justin C. Reis ${ }^{1}$, Xavier Soler*2 \\ ${ }^{1}$ Department of Pulmonary \& Critical Care Medicine, San Antonio Military Medical Center, San Antonio, TX, USA \\ ${ }^{2}$ Division of Pulmonary, Critical Care and Sleep Medicine, University of California San Diego, San Diego, CA, USA
}

Received: February 3, 2016

DOI: $10.5430 /$ crim.v3n2p22
Accepted: March 21, 2016

Online Published: March 30, 2016

\begin{abstract}
Chylothorax is an under recognized cause of pleural effusion, especially when not associated with trauma or malignancy or when the fluid is not milky in appearance. Without proper recognition, chylothorax can lead to significant morbidity and mortality. We present the case of a 77-year-old female with a large intra-abdominal cystic mass. A right pleural effusion was present on a pre-operative computed tomography scan. After laparotomy and resection, the mass was identified as a benign ovarian cystadenoma, and a small-bore chest tube was placed. The effusion was initially exudative, but transitioned to a transudate. Further workup revealed the presence of chylomicrons and a diagnosis of chylothorax was made. The patient had secondary complications including severe lower extremity lymphedema, right hemi-diaphragm elevation, and stenosis of the intrahepatic inferior vena cava. She also had cirrhosis, anasarca, and concomitant severe aortic stenosis. The patient opted to be discharged on hospice care and passed away a week later. Chylothorax should be considered in any patient despite clear pleural effusion. Large abdominal masses may cause chylothorax and should be part of the differential diagnosis.
\end{abstract}

Key Words: Chylothorax, Pleural effusion, Complication, Abdominal mass

\section{INTRODUCTION}

Chylothorax is an under recognized cause of pleural effusion. It results from chylous fluid in the pleural space, usually from a disrupted thoracic duct or one of its many tributaries. The thoracic duct is part of the lymphatics, a complex collecting system parallel with the venous circulation that transports non-soluble vitamins, emulsified fats, and free fatty acids, among other components. ${ }^{[1,2]}$ With surrounding lymphatic vessels, it carries about $100 \mathrm{ml} /$ hour of lymph. Surgical procedures and trauma (e.g. motor vehicle accident) account for the majority of cases of chylothorax. However, non-traumatic causes are also common. ${ }^{[3,4]}$ The fluid is classically milky in appearance in about half of the cases, which usually prompts the diagnosis, although it can be missed when this typical characteristic is not present. Treatment includes removal of the fluid, maintenance of nutrition, prevention of dehydration, and reduction of chyle formation. Furthermore, several interventions or treatment modalities, including surgery, may be necessary. ${ }^{[5]}$ Even with suggested treatment strategies, chylous effusions are a serious condition with significant morbidity and mortality.

\section{Case presentation}

A 77-year-old Hispanic female with a past medical history of systemic hypertension developed increasing abdominal distension and significant lower extremity edema over a two-

${ }^{*}$ Correspondence: Xavier Soler, MD, PhD; Email: xsoler@ucsd.edu; Address: Division of Pulmonary, Critical Care and Sleep Medicine, University of California San Diego, CA, USA. 
year period. Physical examination demonstrated no acute distress, heart rate 103 beats per minute, respiratory rate 18 respirations per minute, blood pressure 104/48, and temperature $36.8^{\circ} \mathrm{C}$. Lung examination revealed dullness to percussion and decreased breath sounds in the right lower field with inspiratory crackles in the lower left area. Cardiac examination demonstrated a $4 / 6$ systolic murmur in the right upper sternal border. Abdominal examination revealed a nontender, distended abdomen with decreased bowel sounds and absence of a fluid wave. Lower extremities demonstrated bilateral elephantiasis with 4+ pitting edema from the proximal thigh to feet. Computed tomography (CT) of the abdomen revealed a $28 \mathrm{~cm} \times 25 \mathrm{~cm} \times 21 \mathrm{~cm}$ multi-septated mass producing compression on intra-abdominal organs, including vertical displacement of the patient's liver and right hemidiaphragm (see Figure 1). She was discharged after undergoing a fine needle aspiration of the mass and elective surgery was planned after pathologic report of ovarian endometrioid cystadenofibroma and a synchronic stage 1 endometrial carcinoma was found. However, she was re-admitted within two weeks for bacteremia (Pseudomonas Aeruginosa). A new chest CT scan demonstrated a large right pleural effusion (see Figure 2) and she was treated with ceftazidime. The patient had a hysterectomy and salpingoopherectomy to remove the giant mass. The patient was found to have severe aortic stenosis (aortic valve area of $0.62 \mathrm{~cm}^{2}$ ), liver cirrhosis likely from long-standing hepatic congestion with hypoalbuminemia, and associated intrahepatic inferior vena cava (IVC) stenosis related to the mass. A 14F bore chest tube was placed for the persistent large right-sided pleural effusion. Baseline pleural chemistries revealed LDH 160 IU/L, pleural fluid protein $1.2 \mathrm{~g} / \mathrm{dl}$, glucose $206 \mathrm{mg} / \mathrm{dl}$, and $\mathrm{pH}$ of 7.56. Cell count and differential showed $378 \mathrm{RBCs}$ and 513 WBCs (71\% neutrophils, 6\% lymphocytes, and 20\% macrophages). Serum total protein and LDH were $5.2 \mathrm{~g} / \mathrm{dl}$ and $132 \mathrm{IU} / \mathrm{L}$, respectively. These results were consistent with an exudative effusion based on Light's criteria. ${ }^{[6]}$ Fluid cultures were negative. Cytology was negative for malignancy. The chest tube output averaged nearly 2 L per day. Repeated fluid analyses including cultures were performed on day 38. At that time the effusion was a transudate with protein serum $v s$. pleural effusion of 0.3 , pleural LDH of 79 IU/L and ratio of 0.4 . Pleural fluid glucose was $203 \mathrm{mg} / \mathrm{dl}$ and triglycerides were $78 \mathrm{mg} / \mathrm{dl}$. Lipoprotein electrophoresis revealed the presence of chylomicrons and a diagnosis of chylothorax was made. A surgical procedure (pleuroperitoneal shunt) was requested but not performed due to the patient's poor functional and nutritional status. Despite aggressive fluid balance therapy, endovascular dilatation of the intrahepatic IVC stricture, and supportive care the patient's condition deteriorated. The patient elected to be transferred to a palliative care facility where she passed away.

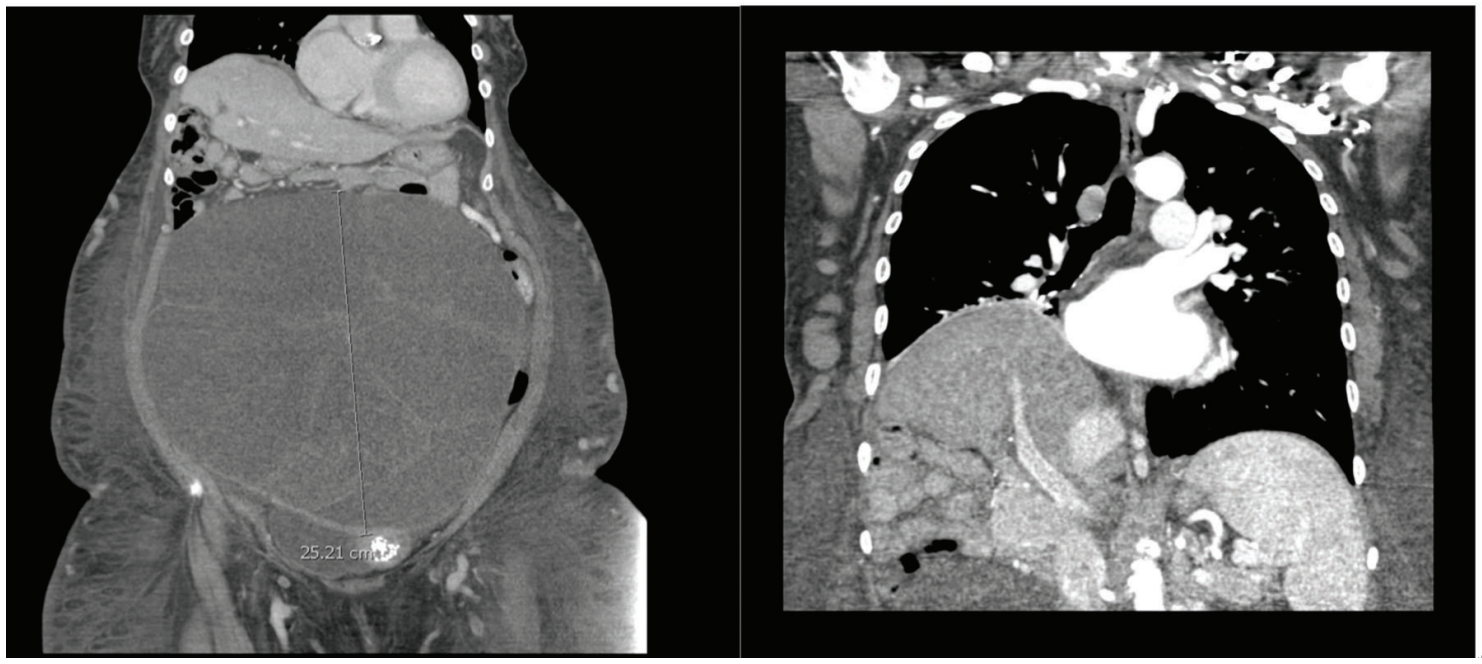

Figure 1. CT Abdominal scan of the patient: Left panel shows the giant $28 \mathrm{~cm} \times 25 \mathrm{~cm} \times 21 \mathrm{~cm}$ mass. Right panel, 1 day post mass removal. Notice the persistent elevated right structures such as diaphragm and liver

\section{DISCUSSION}

This case illustrates a very unusual presentation of chylothorax with transudative fluid lacking a milky appearance and, to our knowledge, the first reported case in which a giant slowgrowing mass dramatically compressed abdominal structures towards the thoracic cavity. The normal peritoneal to tho- racic cavity lymphatic circulation was affected producing elephantiasis and chylous pleural effusion that substantially increased after abdominal surgery to remove the mass.

The most common causes of chylothorax are trauma and malignancy. A case series from the Mayo Clinic of 203 chy- 
lothoraces reported that about half were secondary to trauma while surgical procedures accounted for the majority due to iatrogenic disruption of the ductal system in the thoracic cavity. ${ }^{[3]}$ Almost any thoracic surgery has been reported as a cause of ductal injury due to direct disruption of the thoracic duct or its tributaries. Esophagectomy, pulmonary resection, and congenital heart surgery repair carry considerable risk. Additionally, neck or abdomen surgery can lead to chylothorax as well, although, the risk is considerably less. ${ }^{[4]}$

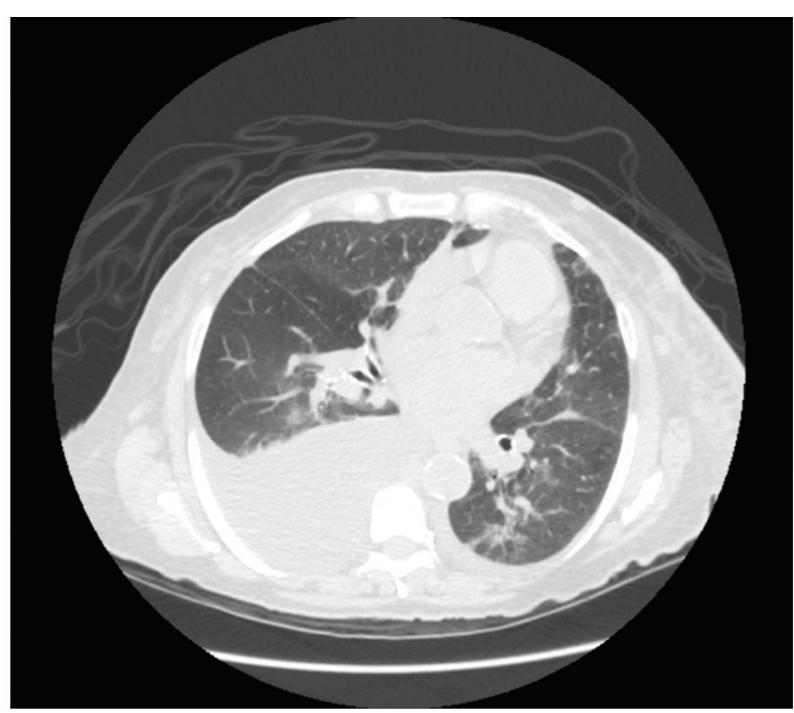

Figure 2. Large pleural effusion day 31 post surgery. A 14F small-bore chest tube was placed and fluid analysed including chylomicrons and triglycerides

In other studies, non-traumatic causes of chylothorax have been reported as high as 72 percent. ${ }^{[2]}$ Lymphoma, chronic lymphocytic leukemia, and metastatic cancer are the leading causes of non-traumatic chylothorax; therefore, a search for the underlying etiology is very important for a chylothorax not related to trauma.

Our patient had long-standing, severely compromised venous return and direct compression of lymphatic structures in the abdomen such the cisterna chyli. ${ }^{[2]}$ Also, she had remarkable diaphragmatic impairment that would make lymphatic reabsorption difficult as the diaphragm participates actively in lymphatic physiology. ${ }^{[2,7]}$ The severe bilateral elephantiasis present on admission confirms the chronic nature of this case. After surgery and removal of the mass, the liver and hemi-diaphragm remained elevated, with persistent impaired lymphatic drainage of the thoracic ductal system. This situation was not improved either after the intrahepatic IVC dilatation of the stricture intended to reduce the gradient between upstream and downstream intrahepatic circulation, which would also have contributed to impaired venous and lymphatic drainage. We did not find common causes of pleural effusion such as left heart failure, hospitalacquired pneumonia, ${ }^{[8]}$ or atelectasis which are prevalent in ICU patients. ${ }^{[9]}$

The diagnosis of chylothorax is typically suspected if the pleural effusion has a milky appearance; however, only about $50 \%$ of chylothoraces have this classic presentation, which is related to high free fatty acid content. ${ }^{[10]}$ Triglyceride levels greater than $110 \mathrm{mg} / \mathrm{dl}$ within the pleural fluid are highly suggestive of chylothorax; however, up to 15 percent may have lower triglycerides levels as in this patient. In these cases, the presence of chylomicrons using lipoprotein electrophoresis will assist in the diagnosis. ${ }^{[10]}$ Additionally, most chylous effusions are exudative by Light's criteria. ${ }^{[6,11]}$ This patient's effusion was initially exudative, but then transitioned to a transudate which is atypical of chylothorax.

Standard treatment includes removal of the fluid, careful maintenance of nutrition, prevention of dehydration, and reduction in the rate of chyle formation. Diet can play an important role in chylothorax management. Often, enteral nutrition containing medium chain triglycerides has been shown to reduce chyle formation, although, parenteral nutrition may be necessary. ${ }^{[12]}$ Somatostatin and octreotide have been used with varying results. The mechanism of action is unknown, but is likely related to decreased lymphatic flow and/or decreased absorption of triglycerides. ${ }^{[13]}$ If a large amount of fluid continues to drain despite a conservative approach, a more invasive procedure may be necessary. ${ }^{[5,14]}$ Pleuroperitoneal shunt, percutaneous transabdominal thoracic duct blockage, pleurodesis via chest tube, thoracoscopy with talc insufflations, and ligation of the thoracic duct through thoracotomy or thoracoscopy have been used for more difficult to manage cases with variable rates of success. ${ }^{[5]}$ Patients are often deemed not suitable candidates for surgery due to their underlying disease process, comorbidities, or significant malnutrition. ${ }^{[4,5,15]}$ Thoracic duct embolization could be a preferred method for addressing post-traumatic or post-surgical chylothoraces. ${ }^{[14]}$

This case is novel because it was initiated by a large abdominal mass and had no-milky appearance, reinforcing the message that non-milky fluid with normal triglycerides can still be a chylothorax. It is important to note that high output pleural effusions ( $>2$ liter/day) should raise suspicion for the presence of chylothorax. In the absence of malignancy, lipoprotein analysis (chylomicrons) is necessary to to avoid delay in specific treatment.

\section{Conclusion}

Pleural effusions are very common. The presence of a rapidly developing pleural fluid collection with high output or the 
presence of previous thoracic and/or abdominal trauma with or without surgery should raise suspicion of a chylothorax despite transudative characteristics or absence of a typical milky appearance. This is important because of specific treatment implications. However, after being diagnosed and treated, the presence of chylothorax is associated with high morbidity and mortality and, therefore, further research is encouraged in this area.

\section{ACKNOWLEDGEMENTS}

We would like to thank Drs. Atul Malhotra, Andrew L Ries, and Laurea Molins Lopez-Rodo for their manuscript revision. We would like to thank Dr. Thomas Griffiths for his assistance with data and image acquisition.

\section{CONFLicts OF INTEREST Disclosure}

The authors have declared no conflicts of interest.

\section{REFERENCES}

[1] Skandalakis JE, Skandalakis LJ, Skandalakis PN. Anatomy of the lymphatics. Surg Oncol Clin N Am. 2007; 16: 1-16. PMid:17336233 http://dx.doi.org/10.1016/j.soc.2006.10.006

[2] Valentine VG, Raffin TA. The management of chylothorax. Chest. 1992; 102: 586-91. http://dx.doi.org/10.1378/chest.102 .2 .586

[3] Doerr CH, Allen MS, Nichols FC, et al. Etiology of chylothorax in 203 patients. Mayo Clinic Proceedings. 2005; 80: 867-70. PMid:16007891 http://dx.doi.org/10.4065/80.7.867

[4] Zabeck H, Muley T, Dienemann H, et al. Management of chylothorax in adults: when is surgery indicated? The Thoracic and Cardiovascular Surgeon. 2011; 59: 243-6. PMid:21425049 http: //dx.doi.org/10.1055/s-0030-1250374

[5] Schild HH, Strassburg CP, Welz A, et al. Treatment options in patients with chylothorax. Deutsches Arzteblatt International. 2013; 110: 819-26. PMid:24333368

[6] Light RW, Macgregor MI, Luchsinger PC, et al. Pleural effusions: the diagnostic separation of transudates and exudates. Ann Intern Med. 1972; 77: 507-13. PMid:4642731 http://dx.doi.org/10. 7326/0003-4819-77-4-507

[7] Abu-Hijleh MF, Habbal OA, Moqattash ST. The role of the diaphragm in lymphatic absorption from the peritoneal cavity. J Anat. 1995; 186 (Pt 3): 453-67. PMid:7559120

[8] Marel M, Zrustova M, Stasny B, et al. The incidence of pleural effusion in a well-defined region. Epidemiologic study in cen- tral Bohemia. Chest. 1993; 104: 1486-9. PMid:8222812 http: //dx.doi.org/10.1378/chest.104.5.1486

[9] Mattison LE, Coppage L, Alderman DF, et al. Pleural effusions in the medical ICU: prevalence, causes, and clinical implications. Chest. 1997; 111: 1018-23. PMid:9106583 http://dx.doi.org/10.13 78/chest.111.4.1018

[10] Staats BA, Ellefson RD, Budahn LL, et al. The lipoprotein profile of chylous and nonchylous pleural effusions. Mayo Clinic Proceedings. 1980; 55: 700-4. PMid:7442324

[11] Maldonado F, Hawkins FJ, Daniels CE, et al. Pleural fluid characteristics of chylothorax. Mayo Clinic Proceedings. 2009; 84: 129-33. PMid:19181646 http://dx.doi.org/10.4065/84.2.129

[12] McGrath EE, Blades Z, Anderson PB. Chylothorax: aetiology, diagnosis and therapeutic options. Respiratory Medicine. 2010; 104: 1-8. PMid:19766473 http://dx.doi.org/10.1016/j.rmed.20 09.08 .010

[13] Kalomenidis I. Octreotide and chylothorax. Current Opinion in Pulmonary Medicine. 2006; 12: 264-7. PMid:16825878 http: //dx.doi.org/10.1097/01.mcp.0000230629.73139.26

[14] Johnson OW, Chick JF, Chauhan NR, et al. The thoracic duct: clinical importance, anatomic variation, imaging, and embolization. Eur Radiol. 2015. http://dx.doi .org/10.1007/s00330-015-411 $2-6$

[15] Paul S, Altorki NK, Port JL, et al. Surgical management of chylothorax. The Thoracic and Cardiovascular Surgeon. 2009; 57: 226-8. PMid:19670117 http://dx.doi.org/10.1055/s-0029-11854 57 